\title{
Variações temporais na estrutura em fitofisionomias de Cerrado e Floresta Estacional Semidecidual em Curvelo, MG
}

\author{
Temporary variations in the structure in phytophysionomies of Cerrado and \\ Semidecidual State Forest in Curvelo, Minas Gerais state
}

\author{
Leovandes Soares da Silva', Thiago José Ornelas Otoni" ${ }^{\mathrm{II}}$, Arthur Duarte Vieira ${ }^{\mathrm{II}}$, \\ Anne Priscila Dias Gonzaga ${ }^{I I I}$, Luciana Botezelli' ${ }^{I V}$, Milton Serpa de Meira Junior ${ }^{\mathrm{V}}$, \\ Israel Marinho Pereira $^{\mathrm{VI}}$, Evandro Luiz Mendonça MachadovI
}

\begin{abstract}
Resumo
Este estudo teve como objetivo analisar a dinâmica da estrutura de espécies arbóreas e relacionar as mudanças com as condições edáficas em fitofisionomias de cerrado stricto sensu (de 2010 a 2015), cerradão (de 2010 a 2015) e floresta estacional semidecidual (de 2011 a 2015) em Curvelo, Minas Gerais. O inventário florestal considerou amostragens do tipo sistemática em 15 unidades amostrais de $20 \times 50$ metros no cerrado stricto sensu (CSS), 10 unidades amostrais $20 \times 50 \mathrm{~m}$ no cerradão $(C D), C A S \geq 15,7 \mathrm{~cm})$ e 25 unidades amostrais de $10 \times 40 \mathrm{~m}$ na floresta estacional semidecidual $(\mathrm{FES}-\mathrm{CAP} \geq 15,7 \mathrm{~cm})$. Amostras de solo foram coletadas em todas as unidades amostrais para caracterização química e granulométrica. Nas três fitofisionomias, o número de indivíduos ingressantes foi inferior ao de egressos. No CSS, a área basal no inventário final foi superior à área basal inicial $\left(23,675-25,845 \mathrm{~m}^{2} / \mathrm{ha}\right)$, devido ao incremento expressivo dos sobreviventes. Ocorreram reduções na área basal no CD $\left(19,322-17,008 \mathrm{~m}^{2} / \mathrm{ha}\right)$ e na FES $\left(28,183-28,148 \mathrm{~m}^{2} /\right.$ ha), decorrente da alta taxa de mortalidade. Nas três fitofisionomias, a mortalidade foi maior nas primeiras classes diamétricas. De forma lenta, o CSS e CD estão se recuperando pós-distúrbio (fogo), estão surgindo novos indivíduos (recrutas) e crescimento diamétrico dos sobreviventes. Durante o intervalo de 5 anos, não foi possível identificar a recuperação do número de indivíduos, enquanto que a FES sem distúrbio do fogo, a perda de indivíduos pela mortalidade e a inclusão de novos indivíduos pelo recrutamento foram mais equilibradas.
\end{abstract}

Palavras-chave: Inventário contínuo; Dinâmica florestal; Variáveis edáficas; Fogo

\begin{abstract}
This study aimed to analyze the structural dynamics of tree species and to relate changes with edaphic conditions in the phytophysiognomies of cerrado stricto sensu (from 2010 to 2015), cerradão (from 2010 to 2015) and seasonal semideciduous forest (from 2011 to 2015) in Curvelo, state of Minas Gerais, Brazil. Forest inventories involved systematic sampling in 15 sample units $(20 \times 50 \mathrm{~m})$ in cerrado strict sensu $(\mathrm{CSS}), 10$ sample units $(20 \times 50 \mathrm{~m})$ in cerradão $(C D ; C A S \geq 15.7 \mathrm{~cm})$ and 25 sample units $(10 \times 40 \mathrm{~m})$ in semideciduous seasonal forest $(S S F ; C A P \geq 15.7 \mathrm{~cm})$. Soil samples were collected in all sample units for the chemical and granulometric characterization. The number of individuals entering was lower than the total recruitment in all phytophysiognomies. Basal area in the final inventory of CSS was higher than initial basal area $\left(23.675-25.845 \mathrm{~m}^{2} / \mathrm{ha}\right)$, due to a significant increase in the surviving individuals. There were reductions in the basal area for DC $\left(19.322-17.008 \mathrm{~m}^{2} / \mathrm{ha}\right)$ and SSF $\left(28.183-28.148 \mathrm{~m}^{2} / \mathrm{ha}\right)$, due to high mortality rates. The mortality was higher for the first diametric classes in all three phytophysiognomies. Individual recruitment and diametric growth of survivors during post fire disturbance recovery was slow for CSS and CD. The number of individuals recovering during a 5-year interval could not be determined, however, without fire disturbance, the loss of individuals due to mortality and the recruitment of new individuals in FES were more balanced.
\end{abstract}

Keywords: Continuous inventory; Forest dynamics; Edaphic variables; Fire.

\footnotetext{
Engenheiro Florestal, Doutorando do Programa de Pós-Graduação em Ciência Florestal, Universidade Federal dos Vales do Jequitinhonha e Mucuri, Campus JK-UFVJM, Rodovia MGT 367 - Km 583, 5.000, Alto da Jacuba, CEP 39100-000, Diamantina (MG), Brasil. leovandessoares@bol. com.br (ORCID: 0000-0002-1609-1010)

Engenheiro Florestal, Me., Departamento de Engenharia Florestal, Universidade Federal dos Vales do Jequitinhonha e Mucuri - UFVJM, Rod. MGT 367 - Km 583, 5.000, Alto da Jacuba, CEP 39100-000, Diamantina (MG), Brasil. tj.otoni@gmail.com (ORCID: 0000-0001-5584-1497) / arthur. duartevieira@gmail.com (ORCID: 0000-0002-0662-504X)

III Bióloga, Dra., Professora da Faculdade de Humanidades da Universidade Federal dos Vales do Jequitinhonha e Mucuri - UFVJM, Rod. MGT 367 - Km 583, 5.000, Alto da Jacuba, CEP 39100-000, Diamantina (MG), Brasil. diaspri@gmail.com (ORCID: 0000-0001-9360-6498)

IV Engenheira Florestal, Dra., Professora do Departamento de Ciência e Tecnologia, Universidade Federal de Alfenas - UNIFAL, CEP 37130-001, Poços de Caldas (MG), Brasil. luciana.botezelli@gmail.com (ORCID: 0000-0002-5916-0442)

Engenheiro Florestal, Dr., Departamento de Engenharia Florestal, Universidade de Brasília - UnB, Campus Darcy Ribeiro, Asa Norte, Caixa Postal 04357, CEP 70910-900, Brasília (DF), Brasil. milton.serpa@gmail.com (ORCID: 0000-0003-2957-8132)

v1 Engenheiro Florestal, Dr., Professor do Departamento de Engenharia Florestal, Universidade Federal dos Vales do Jequitinhonha e Mucuri UFVJM, Rod. MGT 367 - Km 583, 5.000, Alto da Jacuba, CEP 39100-000, Diamantina (MG), Brasil. imarinhopereira@gmail.com (ORCID: 0000-00031035-1253) / machadoelm@gmail.com (ORCID: 0000-0002-9301-5257)
} 


\section{Introdução}

Os fragmentos florestais estão sujeitos a diferentes impactos sejam eles naturais ou antrópicos (APPOLINÁRIO; OLIVEIRA-FILHO; GUILHERME, 2005). A expansão das diferentes culturas agrícolas é uma ameaça constante, pois tem levado à devastação das florestas semideciduais na região do cerrado, restando apenas fragmentos isolados (SILVA; ARAÚJO, 2009).

No Bioma Cerrado, além dos desmatamentos para inserção das culturas agrícolas, outro fator de perturbação é o fogo (KLINK; MACHADO, 2005). No entanto, muitas das espécies representantes das fisionomias do Cerrado são naturalmente adaptadas a esse agente perturbador (HOFFMANN et al., 2012).

A incidência das queimadas contribui para redução da quantidade de indivíduos, isso causa mudanças na estrutura das populações. Segundo Hoffmann et al. (2009), essas mudanças são reflexo tanto da exclusão quanto da incidência das queimadas.

Conforme Chazdon et al. (2007), existe uma complexa interação entre a regeneração natural e os agentes de perturbação. Eventos de perturbação natural (queda e morte de árvores) causam mudanças nas condições de vida, principalmente na disponibilidade de luz e no meio físico. Esses eventos refletem diretamente na densidade, na morte e nos recrutamentos dos indivíduos das diferentes espécies (DELCAMP et al., 2008).

Portanto, estudos relacionados a mudanças temporais em populações vegetais são essenciais, pois por meio desses é possível avaliar as mudanças que ocorrem na estrutura das comunidades vegetais ao longo do tempo. Pelo monitoramento em unidades amostrais permanentes, é possível detectar as possíveis mudanças nos processos transformadores dos fragmentos florestais (HIGUCHI et al., 2008). Esses estudos se baseiam na análise e na interpretação dos dados de recrutamento, mortalidade e aumento ou redução na área basal, com a função de identificar as mudanças estruturais das comunidades (MAGALHÃES et al., 2017).

Dessa maneira, objetivou-se neste trabalho analisar a relação das variáveis ambientais edáficas na dinâmica estrutural de espécies arbóreas, em distintas fitofisionomias de cerrado e de floresta estacional semidecidual, que ocorreram em um intervalo de cinco anos de medição (2010 e 2015).

\section{Material e métodos}

Este estudo foi desenvolvido na fazenda do Moura, localizada em Curvelo, Minas Gerais. A fazenda possui área aproximada de 436 hectares (ha), encontra-se localizada nas coordenadas geográficas $18^{\circ} 49^{\prime} 26.12^{\prime \prime} \mathrm{S}, 44^{\circ} 24^{\prime} 12.69^{\prime \prime}$ O e sobre uma altitude média de 715 metros. O clima da região é do tipo Aw tropical - segundo a classificação de Koppen, temperatura média de $28^{\circ} \mathrm{C}$, índice pluviométrico anual de 1200 mm, concentrados no verão (STRAHLER, 2002).

Na propriedade são encontradas três distintas fitofisionomias: cerrado stricto sensu (CSS), cerradão (CD) e floresta estacional semidecidual (FES).

O CSS se encontra nas coordenadas $18^{\circ} 50^{\prime} 7.26^{\prime \prime} \mathrm{S}, 44^{\circ} 23^{\prime} 33.49^{\prime \prime O}$ e possui 54 ha de área onde uma estrada percorre o interior do fragmento. O solo é do tipo Latossolo vermelho distrófico (EMBRAPA, 2004). O estrato herbáceo é composto por gramíneas naturais no interior e gramíneas exóticas nas bordas do fragmento.

O CD se encontra nas coordenadas $18^{\circ} 49^{\prime} 56.11^{\prime \prime} \mathrm{S}, 44^{\circ} 23^{\prime} 6.42^{\prime \prime} \mathrm{O}$ e possui aproximadamente 80,0 ha sobre relevo aplainado e solo classificado como Latossolo vermelho distrófico (EMBRAPA, 2004). Os fragmentos CSS e CD foram atingidos por um incêndio em toda sua extensão após o inventário 2010 (OTONI et al., 2013) e, posteriormente, não foi registrado novo indício de incêndio.

Já a FES se encontra nas coordenadas $18^{\circ} 49^{\prime} 21.17^{\prime \prime S}, 44^{\circ} 23^{\prime} 55.91^{\prime \prime O}$ e possui aproximadamente 162 ha, seu estágio sucessional é secundário e se encontra entre as 
fitofisionomias do CSS e CD e por áreas modificadas pela agricultura. Há histórico de perturbação antrópica, como retirada esporádica de madeira nos últimos quinze anos nas bordas da floresta. Não houve registros de retirada de madeira nas parcelas, sendo registrada apenas queda de árvores causada pelo vento e poucas trilhas de gado no interior da floresta.

\section{Amostragem da vegetação}

As fitofisionomias do CSS e CD foram inventariadas no ano de 2010 por Otoni et al. (2013), onde o autor instalou 15 unidades amostrais permanentes de $20 \times 50 \mathrm{~m}$ no CSS e 10 unidades amostrais permanentes de $20 \times 50 \mathrm{~m}$ no CD, distribuídas de maneira sistemática a cada $100 \mathrm{~m}$. Dentro das unidades amostrais, todos os indivíduos com circunferência à altura do solo (CAS) com $\geq 15,7 \mathrm{~cm}$ foram identificados e tiveram seus diâmetros mensurados. Indivíduos com caules múltiplos foram mensurados quando a raiz da soma dos quadrados obedeceu ao critério de inclusão $C A S \geq 15,7 \mathrm{~cm}$.

A FES foi inventariada no ano de 2011 por Franco (2012), onde o autor instalou 25 unidades amostrais permanentes com dimensões de $10 \times 40 \mathrm{~m}$ distanciadas $30 \mathrm{~m}$ entre si. Em cada unidade amostral, os indivíduos vivos com circunferência à altura do peito $(C A P) \geq 15,7 \mathrm{~cm}$ foram identificados e tiveram seus diâmetros mensurados. Foram fixadas placas numeradas nos indivíduos que alcançaram o diâmetro estabelecido, para serem monitorados.

A identificação das espécies foi realizada conforme descrito por Franco (2012) e Otoni et al. (2013). Assim, foram coletadas exsicatas e posteriormente levadas para a identificação, sendo esta realizada por pesquisas na literatura, por especialistas, ou por comparações com espécimes armazenadas no Herbário Dendrológico Jeanine Felfili (HDJF) da Universidade Federal dos Vales do Jequitinhonha e Mucuri (UFVJM). A classificação botânica seguiu o sistema APG IV (ANGIOSPERM PHYLOGENY GROUP, 2016). O nome das espécies foi atualizado usando a Lista de Espécies da Flora do Brasil 2020.

O presente estudo reavaliou as medições dos dois inventários em 2015, utilizando o mesmo critério amostral. Os sobreviventes foram mensurados novamente; os mortos, registrados. Os indivíduos ingressantes (recrutas) foram identificados e tiveram placas fixadas com numeração e coletadas as informações de diâmetro.

\section{Variáveis do solo}

Para análise química e granulométrica, foram coletadas cinco amostras compostas de solo (500 gramas) em cada unidade amostral (camada de 0 a $20 \mathrm{~cm}$ de profundidade). As análises foram realizadas no Laboratório de Análise de Solos da (UFVJM), conforme o protocolo da EMBRAPA (1997).

\section{Variável fogo}

Na coleta da variável fogo, foram selecionados 10 indivíduos por unidade amostral do CSS e CD atingidos pelo incêndio. Em seguida, com auxílio de uma trena, foram coletadas as alturas de crestamento dos indivíduos selecionados. Calcularam-se as médias da altura de crestamento por unidade amostral (Metodologia dos próprios autores).

\section{Análise da dinâmica estrutural}

Calcularam-se os parâmetros de dinâmica para amostra total, realizados por meio da demografia (contagem) de indivíduos arbóreos e área basal. Foi assumida a soma das áreas seccionais à altura do peito e assumiu-se uma relação circular com o CAS para o CSS, CD e CAP para FES. 
Por meio do modelo exponencial proposto por Sheil, Jennings e Savill (2000), assim foram, portanto, calculadas as taxas médias anuais dos mortos $(\mathrm{M})$ e recrutas $(\mathrm{R})$, baseando na quantidade de indivíduos, e as médias anuais de perda $(\mathrm{P})$ e ganho $(\mathrm{G})$ de área basal. Por esse modelo, foram assumidas mudanças do tamanho da população por espaço de tempo com proporção constante ao tamanho inicial da população. Para isso, foram usadas as seguintes equações, onde:

$$
\begin{array}{lr}
M=\left\{1-\left[\left(N_{0}-m\right) / N_{0}\right]^{1 / t} \times 100 ;\right. & R=\left[1-\left(1-r / N_{f}\right]^{1 / t} \times 100 ;\right. \\
P=\left\{1-\left[\left(A B 0-A B_{m} A_{d}\right) / A B_{0}\right]^{1 / t}\right\} \times 100 ; & G=\left\{1-\left[1-\left(A_{r}+A B_{g}\right) / A B_{f} f^{1 / t}\right\} \times 100 ;\right.
\end{array}
$$

Em que: $\mathrm{N}_{0}$ e $\mathrm{N}_{\mathrm{f}}=$ número de indivíduos, inventário inicial e final respectivamente; $\mathrm{t}$ = tempo entre inventário inicial e final, respectivamente; $\mathrm{AB}_{0}$ e $\mathrm{AB}_{\mathrm{f}}=$ áreas basais dos indivíduos nos dois inventários; $\mathrm{AB}_{\mathrm{m}}$ e $\mathrm{AB}_{\mathrm{r}}=$ área basal dos mortos e ingressantes, respectivamente, e $A B_{\mathrm{d}}-\mathrm{AB}_{\mathrm{g}}=$ área basal do decremento e incremento.

A partir das médias das taxas dos mortos, recrutas, perda e ganho, obtiveram-se as taxas de rotatividade (turnover) em número de indivíduos (TN) e área basal (TAB). Isso foi para expressar a mudança global, conforme Korning e Balslev (1994) e Oliveira-Filho, Mello e Scolforo (1997).

$$
\mathrm{TN}=(\mathrm{M}+\mathrm{R}) / 2 ; \quad \mathrm{TAB}=(\mathrm{P}+\mathrm{G}) / 2 ;
$$

Em que: $\mathrm{M}=$ taxa anual dos indivíduos mortos; $\mathrm{R}$ = taxa anual dos indivíduos recrutas e $\mathrm{P}$ e $\mathrm{G}=$ taxa de perda e ganho respectivamente.

Foram também calculadas as taxas de mudanças líquidas realizadas conforme Korning e Balslev (1994), para área basal $\left(\mathrm{Ch}_{\mathrm{AB}}\right)$ e para número de árvores $(\mathrm{ChN})$.

$$
\mathrm{Ch}_{\mathrm{AB}}=\left[\left(\mathrm{AB}_{\mathrm{f}} / \mathrm{AB}_{0}\right) 1 / \mathrm{t}-1\right] \times 100 ; \quad \mathrm{Ch}_{\mathrm{N}}=\left[\left(\mathrm{N}_{\mathrm{f}} / \mathrm{N}_{0}\right) 1 / \mathrm{t}-1\right] \times 100
$$

Em que: $\mathrm{t}$ = tempo entre o inventário inicial e final, respectivamente; $\mathrm{N}_{0}$ e $\mathrm{N}_{\mathrm{f}}=$ número de indivíduos inventário inicial e final, respectivamente, e $\mathrm{AB}_{0}$ e $\mathrm{AB}_{\mathrm{f}}=$ áreas basais dos indivíduos primeiro e segundo inventário.

\section{Mudanças nas classes diamétricas}

Conforme estudos de dinâmica florestal realizados por Appolinário, Oliveira-Filho e Guilherme (2005), na dinâmica dos indivíduos por classes diamétricas, empregaram-se intervalos de classes crescentes, para diminuir o forte decréscimo dos indivíduos nas maiores classes de diâmetros.

Para interpretação das mudanças por classe de diâmetro, foram contados os indivíduos que permaneceram na classe, mortos, recrutas, imigração (ingrowth) e emigração (outgrowth) na classe. A imigração e emigração podem ser progressivas ou regressivas (LIEBERMAN et al.,1985). Mediante comparações entre contagens de Poisson, foram assim calculadas as diferenças entre número de ingressos (recrutas + imigrantes) e indivíduos egressos (mortos + emigrantes) para cada classe de diâmetro por fitofisionomia.

\section{Processamento dos dados}

As correlações de Pearson foram empregadas usando combinações entre as variáveis de dinâmica incluindo área basal dos mortos, recrutas e incremento, sendo estas correlacionadas com as variáveis edáficas e variável fogo para o CSS e CD. As análises de correlação foram realizadas por meio do software BioEstat 5.3 (AYRES et al., 2007). 


\section{Resultados e discussão}

No decorrer de cinco anos, foi registrado o decréscimo de 184 indivíduos e de uma espécie (62) no CSS. Nesse mesmo período, o CD apresentou a diminuição de 810 indivíduos, 10 espécies, quatro famílias e incremento de uma nova espécie. Por fim, a FES houve a diminuição de 82 indivíduos arbóreos, três espécies e (uma família), mas com o saldo de duas novas espécies (Tabela 1).

Tabela 1 - Mudanças estruturais em diferentes fitofisionomias: Cerrado stricto sensu = CSS, Cerradão $=$ CD e Floresta Estacional Semidecidual $=$ FES, localizados na Fazenda do Moura em Curvelo, Minas Gerais

Table 1 - Structural changes in different phytophysiognomies: Cerrado stricto sensu = CSS, Cerradão $=$ CD and Semideciduous Seasonal Forest $=$ FES, located at 'Fazenda do Moura' in Curvelo, Minas Gerais state

\begin{tabular}{|c|c|c|c|}
\hline Fitofisionomias & CSS & CD & FES \\
\hline Número de unidade amostral & 15 & 10 & 25 \\
\hline \multicolumn{4}{|l|}{ Número de famílias } \\
\hline Inicial & $26^{1}$ & $34^{1}$ & $43^{2}$ \\
\hline Final & 26 & 30 & 42 \\
\hline \multicolumn{4}{|l|}{ Número de espécies: } \\
\hline Inicial & $63^{1}$ & $100^{1}$ & $132^{2}$ \\
\hline Final & 62 & 90 & 129 \\
\hline \multicolumn{4}{|l|}{ Número de árvores } \\
\hline Inicial (número de indivíduos) & $3094^{1}$ & $2440^{1}$ & $1107^{2}$ \\
\hline Final (número de indivíduos) & 2940 & 1630 & 1025 \\
\hline Mortas (número de indivíduos) & 317 & 904 & 134 \\
\hline Recrutas (número de indivíduos) & 163 & 94 & 52 \\
\hline Sobreviventes (número de indivíduos) & 2777 & 1536 & 973 \\
\hline Taxa Mortalidade $\left(\%\right.$ ano- $\left.^{1}\right)$ & 2,13 & 8,84 & 2,54 \\
\hline Taxa Recrutamento $\left(\%\right.$ ano- $\left.^{-1}\right)$ & 1,13 & 1,18 & 1,03 \\
\hline Taxa Rotatividade $\left(\%\right.$ ano- $\left.^{1}\right)$ & 1,63 & 5,01 & 1,80 \\
\hline Taxa Mudança (\% ano- $\left.{ }^{1}\right)$ & $-1,01$ & $-7,75$ & $-1,52$ \\
\hline \multicolumn{4}{|l|}{ Área Basal } \\
\hline Inicial $\left(\mathbf{m}^{2} / \mathbf{h a}\right)$ & $23,675^{1}$ & $19,322^{1}$ & $28,183^{2}$ \\
\hline Final $\left(\mathbf{m}^{2} / \mathbf{h a}\right)$ & 25,845 & 17,008 & 28,148 \\
\hline $\operatorname{Mortas}\left(\mathbf{m}^{2} / \mathbf{h a}\right)$ & 1,587 & 4,863 & 2,682 \\
\hline Decremento dos sobreviventes $\left(\mathbf{m}^{2}\right)$ & $-0,422$ & $-0,202$ & $-0,160$ \\
\hline $\operatorname{Recrutas}\left(\mathbf{m}^{2} / \mathbf{h a}\right)$ & 0,463 & 0,284 & 0,122 \\
\hline Crescimento Sobrevivência $\left(\mathbf{m}^{2} / \mathbf{h a}\right)$ & 25,381 & 16,724 & 28,026 \\
\hline Mudança líquida (\%) & 1,769 & $-2,518$ & $-0,024$ \\
\hline Taxa de perdas $\left(\%\right.$ ano- $\left.^{-1}\right)$ & 1,800 & 5,900 & 2,103 \\
\hline Taxa de ganho $\left(\%\right.$ ano- $\left.^{1}\right)$ & 3,501 & 5,732 & 2,079 \\
\hline
\end{tabular}

Fonte: Autores (2017)

Em que: CSS e CD com CAS $\geq 5 \mathrm{~cm}$ e FES DAP $\geq 5 \mathrm{~cm}$. 1. Realizado em 2010 (Otoni, 2013) 2. Realizado em 2011 (Franco, 2012). 
Conforme a Tabela 1, houve maior redução de indivíduos na comunidade do CD em relação ao CSS e à FES. Houve maior quantidade de recrutas no CSS e menor no CD e na FES. A maior taxa de mortalidade foi no CD em relação ao CSS e à FES. A taxa de mudança foi maior no CD em relação às outras duas fitofisionomias, e a área basal aumentou apenas no CSS, diminuiu no CD e na FES.

Os eventos de fogo e vento ocorridos após o inventário inicial podem ter ocasionado a maior mortalidade de indivíduos nas fitofisionomias de CSS e CD em relação à FES. No entanto, a quantidade de indivíduos mortos no CSS foi inferior à encontrada por Almeida et al. (2014). Os autores perceberam que a quantidade de indivíduos decresceu com a ocorrência das queimadas em relação aos locais protegidos do fogo, que ocorreu aumento do número de indivíduos (SILVA NETO et al., 2017).

A taxa de mortos no CSS foi $2,13 \%$ ano- $^{1}$. Esse valor está dentro do intervalo encontrado em estudos de dinâmica no cerrado, porém tal valor foi inferior aos resultados encontrados por Sato, Gardo e Miranda (1998), que constataram taxas de mortalidade que variaram entre $6,4 \%$ e $13 \%$.

No CSS a rapidez dos processos dinâmicos em número de árvores obtida pela taxa de rotatividade foi de $1,13 \%$ ano- $^{-1}$. A exclusão dos indivíduos decorrente da mortalidade refletiu na área basal final a perda foi de $1,587 \mathrm{~m}^{2} /$ ha, no entanto foi a menor entre as fitofisionomias estudadas. Almeida et al. (2014) afirmaram que o incremento em área basal tende a aumentar sem os distúrbios do fogo no Cerrado.

No CSS o incremento dos sobreviventes junto com os recrutas $3,715 \mathrm{~m}^{2} / \mathrm{ha}^{-1} 0,463 \mathrm{~m}^{2} / \mathrm{ha}^{-1}$ contribuiu para que a área basal final fosse superior a inicial $23,675-25,845 \mathrm{~m}^{2} / \mathrm{ha}$. O decremento foi $-0,422 \mathrm{~m}^{2} / \mathrm{ha}^{-1}$, no entanto a taxa de aumento em área basal foi superior à taxa de perda, isso resultou em mudança líquida positiva. A morte dos indivíduos alterou a estrutura do CSS, no entanto recebeu novos indivíduos recrutados 163, assim a diferença entre a taxa de mortos e recrutas foi de $1 \%$ ano $^{-1}$.

A redução de indivíduos foi mais alta no CD com 904 indivíduos mortos, isso contribuiu para elevar a taxa de mortalidade $8,84 \% \mathrm{ano}^{-1}$. Foram inclusos 94 indivíduos pelo recrutamento, a rotatividade foi de $5,01 \%$ ano- $^{-1}$ (Tabela 1 ). No entanto, o CD demonstrou ser resiliente, pois surgiram novos indivíduos que não foram amostrados neste inventário pelo critério de inclusão e provavelmente deverão ser incluídos na próxima remedição. Foi observado que o CD produz muita matéria morta e, em uma possível ocorrência de incêndio, pode aumentar a quantidade de indivíduos mortos, por isso deve ser protegido do fogo.

As informações na Tabela 1 mostram que a perda de indivíduos no CD resultou na redução de $4,863 \mathrm{~m}^{2} /$ ha na área basal final. Nos dois inventários, a área basal foi de 19,322 $17,008 \mathrm{~m}^{2} /$ ha, respectivamente, no entanto essa diferença não foi mais acentuada, porque os sobreviventes aumentaram significativamente seus diâmetros $4,063 \mathrm{~m}^{2} / \mathrm{ha}^{-1}$, os recrutas somaram $0,284 \mathrm{~m}^{2} / \mathrm{ha}^{-1}$ de área basal, o decremento foi de $-0,202 \mathrm{~m}^{2} / \mathrm{ha}^{-1}$. Ivanauskas, Monteiro e Rodrigues (2003) afirmam que de fato os sobreviventes são favorecidos pela adição de nutrientes acelerados pelo fogo, que aumenta o processo de lixiviação dos minerais da camada de serapilheira, ou seja, após um incêndio, há um aumento na concentração de nutrientes no solo. O crescimento dos sobreviventes compensou a perda de área basal dos mortos, possivelmente a abertura da vegetação, resultado da mortalidade, tenha favorecido o crescimento dos sobreviventes.

Na FES, a estrutura variou pouco no período avaliado, a taxa de recrutamento foi inferior à taxa de mortalidade. Em uma unidade amostral, ocorreu queda de indivíduos provocada naturalmente pelo vento. Nessa unidade amostral, a metade dos indivíduos morreu, isso contribuiu para elevar a taxa de mortalidade. Observou-se também que nessa unidade referida, o surgimento de novos recrutas que não alcançaram o diâmetro mínimo estabelecido neste inventário.

Segundo Machado e Oliveira-Filho (2010), quando se reduz a densidade também se diminui a competição, o que é compensado pelo ganho diamétrico dos sobreviventes. No entanto, 
a perda dos indivíduos interferiu pouco na área basal: no primeiro inventário foi $28,183 \mathrm{~m}^{2} /$ ha e ela diminuiu para $28,148 \mathrm{~m}^{2} /$ ha no segundo inventário. Resultados parecidos foram encontrados por Machado e Oliveira-Filho (2010) e por Mews et al. (2011) em Floresta Estacional Semidecidual, com o mesmo intervalo de tempo empregado neste estudo. Já os resultados encontrados por Figueiredo et al. (2013) mostram que, no primeiro inventário área basal $26,15 \mathrm{~m}^{2} /$ ha foi inferior a $27,51 \mathrm{~m}^{2} /$ ha encontradas no segundo inventário.

Já a mudança líquida sofreu uma leve redução, no entanto foi compensada pelos recrutas e incremento dos sobreviventes. Segundo Machado e Oliveira Filho (2010), a redução de indivíduos também pode ser interpretada como parte do processo de competição entre eles ou do próprio ciclo de vida.

A Tabela 2 mostra que as modificações foram mais intensas nas classes diamétricas iniciais (5 - 10 e $10-20 \mathrm{~cm}$ ), seja pela mortalidade ou pelas mudanças para classes posteriores. Mesmo utilizando intervalos de classes crescentes, a distribuição diamétrica apresentou-se na forma do $J$-invertido nos dois inventários, indicando o surgimento de recrutas.

Os resultados indicam que os indivíduos nos primeiros intervalos de classes mostraram ser menos resistentes ao impacto do fogo e possivelmente à competição, mudanças tanto pelo crescimento para outras classes como pela mortalidade foram maiores nos primeiros intervalos de classes. Isso indica a dificuldade do surgimento e estabelecimento dos recrutas, que pode estar surgindo de maneira lenta. Ribeiro et al. (2012) também notaram mudanças semelhantes nos primeiros intervalos de classes após a passagem de fogo.

No intervalo da primeira classe $(5$ a $10 \mathrm{~cm}$ ) os egressos (emigrantes + mortos) foram superiores aos ingressantes (imigrantes + recrutas). Separadamente, os eventos migratórios (imigrantes + emigração), foram mais intensos no CSS, seguido pelo CD e pela FES, (Tabela 2).

Tabela 2 - Mudanças estruturais em fitofisionomias de Cerrado stricto sensu (CSS), Cerradão (CD) e Floresta estacional semidecidual (FES)

Table 2 - Structural changes in Cerrado stricto sensu (CSS), Cerradão (CD) and Semidecidual seasonal forest (FES)

\begin{tabular}{|c|c|c|c|c|c|c|c|c|c|c|c|}
\hline & \multicolumn{3}{|c|}{ № de árvores } & \multicolumn{3}{|c|}{ Mortas } & \multirow{2}{*}{$\begin{array}{c}\text { Emig. } \\
\text { № } \\
\end{array}$} & \multirow{2}{*}{$\begin{array}{c}\text { Rec. } \\
\text { № }^{\circ} \\
\end{array}$} & \multirow{2}{*}{$\begin{array}{c}\text { Imig. } \\
\text { № } \\
\end{array}$} & \multicolumn{2}{|c|}{ Cont. Poisson } \\
\hline & $1^{\circ} \mathrm{Inv}$ & $2^{\circ} \operatorname{Inv}$ & Esp. & № & Esp. & $\%$ ano $^{-1}$ & & & & Z & $P$ \\
\hline \multicolumn{12}{|l|}{ CSS } \\
\hline 5 a 10 & 2290 & 1975 & 2176,02 & 272 & 234,63 & 2,92 & 221 & 163 & 15 & 12,16 & 0,001 \\
\hline 10 a 20 & 746 & 894 & 708,87 & 43 & 76,43 & 0,98 & 32 & 0 & 223 & 8,57 & 0,001 \\
\hline 20 a 40 & 48 & 59 & 45,61 & 2 & 4,92 & 0,69 & 4 & 0 & 17 & 2,29 & 0,05 \\
\hline 40 a 80 & 10 & 12 & 9,50 & 0 & 1,02 & 0,00 & 0 & 0 & 2 & 1,41 & $\mathrm{~ns}$ \\
\hline Total & 3094 & 2940 & & 317 & & & 257 & 163 & 257 & & \\
\hline \multicolumn{12}{|l|}{$C D$} \\
\hline 5 a 10 & 1664 & 858 & 1111,61 & 772 & 616,50 & 36,87 & 137 & 93 & 10 & 25,34 & 0,001 \\
\hline 10 a 20 & 670 & 618 & 447,58 & 121 & 248,23 & 4,26 & 65 & 1 & 133 & 2,91 & 0,002 \\
\hline 20 a 40 & 100 & 147 & 66,80 & 11 & 37,05 & 1,54 & 0 & 0 & 59 & 5,74 & 0,001 \\
\hline 40 a 80 & 6 & 7 & 4,01 & 0 & 2,22 & 0,00 & 0 & 0 & 1 & 1,00 & $\mathrm{~ns}$ \\
\hline Total & 2440 & 1630 & & 904 & & & 202 & 94 & 203 & & \\
\hline
\end{tabular}


Tabela 2 - Conclusão ...

Table 2 - Conclusion ...

\begin{tabular}{|c|c|c|c|c|c|c|c|c|c|c|c|}
\hline & \multicolumn{3}{|c|}{ № de árvores } & \multicolumn{3}{|c|}{ Mortas } & \multirow{2}{*}{$\frac{\text { Emig. }}{\text { № }}$} & \multirow{2}{*}{$\frac{\text { Rec. }}{\text { № }}$} & \multirow{2}{*}{$\frac{\text { Imig. }}{\text { № }}$} & \multicolumn{2}{|c|}{ Cont. Poisson } \\
\hline & $1^{\circ} \operatorname{Inv}$ & $2^{\circ} \operatorname{Inv}$ & Esp. & № & Esp. & $\%$ ano $^{-1}$ & & & & $Z$ & $P$ \\
\hline \multicolumn{12}{|l|}{ FES } \\
\hline 5 a 10 & 593 & 530 & 549,07 & 83 & 71,78 & 3,35 & 34 & 52 & 2 & 4,82 & 0,001 \\
\hline 10 a 20 & 263 & 257 & 243,52 & 22 & 31,84 & 1,77 & 19 & 0 & 35 & 0,69 & ns \\
\hline 20 a 40 & 206 & 191 & 190,74 & 26 & 24,94 & 2,88 & 6 & 0 & 17 & 2,14 & 0,05 \\
\hline 40 a 80 & 45 & 47 & 41,67 & 3 & 5,45 & 1,31 & 0 & 0 & 5 & 0,71 & ns \\
\hline Total & 1107 & 1025 & & 134 & & & 59 & 52 & 59 & & \\
\hline
\end{tabular}

Fonte: Autores (2017)

Em que: Esp. = Frequências esperadas para o número de indivíduos no segundo inventário; $\mathrm{N}=$ número de indivíduos mortos por classe de diâmetro, baseando-se na distribuição do número de indivíduos por classe de DAS e DAP, do primeiro inventário; Emig. = taxas de mortalidade anual e os números de emigrantes; Rec = recrutas; Imig. = imigrantes, comparações de Poisson entre saídas (mortos + emigrantes) e entradas (recrutas + imigrantes).

O padrão de distribuição diamétrica neste estudo é comum de comunidades consideradas autorregenerativas, como citadas por Lopes, Vale e Shiavini (2009), em que grande parte dos indivíduos está distribuída nos primeiros intervalos de classes, conforme resultados encontrados por Mews et al. (2011) e Almeida et al. (2014).

No primeiro intervalo de classe, a mortalidade ficou acima dos valores esperados. Além disso, houve passagens de indivíduos para classes diamétricas superiores. Resultados semelhantes foram encontrados por Almeida et al. (2014). A chegada de novos indivíduos (recrutas + imigrantes) vindos de outras classes foi maior no segundo intervalo de classe.

Nas maiores classes, as mudanças foram menos acentuadas. No CD e no CSS, os resultados revelam que os indivíduos maiores foram mais resistentes aos distúrbios. Segundo Hoffmann et al. (2012), indivíduos maiores têm mais possibilidades de sobreviver aos distúrbios.

Pela contagem de Poisson, as mudanças dos indivíduos entre as classes diamétricas mostraram que, nos três primeiros intervalos de classes, as mudanças foram significativas, tanto no CD quanto no CSS. Na maior classe, as mudanças foram menos intensas, enquanto na FES as mudanças foram maiores no primeiro e terceiro intervalo de classe e não significativa na segunda e quarta classe, respectivamente. As mudanças foram mais pronunciadas na primeira classe. Provavelmente, a competição é maior entre os indivíduos menores, isso provoca mortalidade entre eles, enquanto os sobreviventes são favorecidos pelo aumento de espaço e disponibilidade de nutrientes, e com o tempo ganham biomassa e mudam para os intervalos de classes maiores. À medida que aumentaram os diâmetros, diminuiu a mortalidade, como observado por Mews et al. (2011).

Já o número de indivíduos ingressantes (emigrantes) foi superior ao de egressos no segundo intervalo de classe no CSS. Alguns estudos mostram que as fitofisionomias de Cerrado protegido das queimadas podem favorecer o adensamento da vegetação (RIBEIRO et al., 2012; ALMEIDA et al., 2014), à medida que reduziu a frequência das queimadas surgiram indivíduos de espécies menos resistentes ao fogo. Portanto, as altas taxas de mortalidade são decorrentes das espécies menos resistentes ao fogo, como, por exemplo, Roupala montana, Styrax ferrugineus e Myrsine guianensis (ALMEIDA et al., 2014; RIOS et al., 2018). Segundo Pivello et al. (2011), as queimadas periódicas são importantes para manutenção estrutural da 
paisagem, função, composição e integridade ecológica do Cerrado. O aumento da frequência das queimadas torna o ambiente mais instável (VASCONCELOS; ARAÚJO; BRUNA, 2014).

Quando feita a análise de correlação de Pearson das variáveis de dinâmica, no CSS as correlações entre indivíduos recrutas e variáveis do solo foram todas não significativas, o mesmo aconteceu entre indivíduos mortos e variáveis do solo. As correlações entre variáveis do solo e área basal dos recrutas e mortos também foram não significativas. Apenas crescimento em área basal correlacionou significativamente, tendo correlações positivas com alumínio, acidez potencial, capacidade de troca de cátions a $\mathrm{pH}$ 7,0 e argila, porém negativas com saturação por bases e Silte (Tabela 3).

Tabela 3 - Correlação entre: (a) o número de indivíduos (recrutas e mortos), (b) área basal dos (recrutas, mortos e incremento) com as variáveis ambientais e do fogo

Table 3 - Correlation between: (a) number of individuals (recruits and dead), (b) basal area of (Recruits, dead and increment) with environmental and fire variables

a)

\begin{tabular}{|c|c|c|c|c|c|c|}
\hline \multirow{3}{*}{ Variáveis de solo } & \multicolumn{6}{|c|}{ Número de indivíduos por Fitofisionomia } \\
\hline & \multirow[t]{2}{*}{ CSS } & CD & FES & CSS & CD & FES \\
\hline & & \multicolumn{2}{|c|}{ Recrutas } & \multicolumn{2}{|c|}{ Mortalidade } & \\
\hline V (\%) & $0,33^{\text {ns }}$ & $0,35^{\mathrm{ns}}$ & $0,16^{\mathrm{ns}}$ & $-0,46^{\mathrm{ns}}$ & $-0,34^{\mathrm{ns}}$ & $-0,05^{\mathrm{ns}}$ \\
\hline $\mathbf{P}\left(\mathbf{m g} / \mathbf{d m}^{3}\right)$ & $0,19^{\text {ns }}$ & $0,10^{\text {ns }}$ & $0,46^{*}$ & $0,09^{\text {ns }}$ & $0,12^{\mathrm{ns}}$ & $-0,23^{\text {ns }}$ \\
\hline $\mathbf{K}\left(\mathbf{m g} / \mathbf{d m}^{3}\right)$ & $-0,26^{\text {ns }}$ & $0,10^{\text {ns }}$ & $0,15^{\mathrm{ns}}$ & $-0,05^{\mathrm{ns}}$ & $0,00^{\text {ns }}$ & $-0,32^{\text {ns }}$ \\
\hline $\mathrm{Ca}\left(\mathbf{c m o l c} / \mathbf{d m}^{3}\right)$ & $0,38^{\mathrm{ns}}$ & $-0,13^{\text {ns }}$ & $0,07^{\mathrm{ns}}$ & $-0,07^{\mathrm{ns}}$ & $0,25^{\mathrm{ns}}$ & $-0,02^{\text {ns }}$ \\
\hline $\operatorname{Mg}\left(\mathbf{c m o l c} / \mathbf{d m}^{3}\right)$ & $-0,26^{\text {ns }}$ & $0,60^{\text {ns }}$ & $-0,19^{\mathrm{ns}}$ & $-0,14^{\mathrm{ns}}$ & $-0,48^{\mathrm{ns}}$ & $0,18^{\mathrm{ns}}$ \\
\hline $\mathrm{Al}\left(\mathbf{c m o l c} / \mathbf{d m}^{3}\right)$ & $-0,31^{\text {ns }}$ & $0,24^{\mathrm{ns}}$ & $0,06^{\mathrm{ns}}$ & $0,13^{\mathrm{ns}}$ & $-0,07^{\mathrm{ns}}$ & $-0,18^{\mathrm{ns}}$ \\
\hline $\mathbf{H}+\mathbf{A l}\left(\mathbf{c m o l c} / \mathbf{d m}^{3}\right)$ & $-0,25^{\mathrm{ns}}$ & $-0,34^{\mathrm{ns}}$ & $-0,26^{\mathrm{ns}}$ & $0,49^{\text {ns }}$ & $-0,40^{\text {ns }}$ & $0,08^{\mathrm{ns}}$ \\
\hline SB $\left(\mathbf{c m o l c} / \mathbf{d m}^{3}\right)$ & $0,12^{\mathrm{ns}}$ & $0,23^{\mathrm{ns}}$ & $0,03^{\mathrm{ns}}$ & $-0,11^{\mathrm{ns}}$ & $-0,11^{\mathrm{ns}}$ & $0,00^{\text {ns }}$ \\
\hline$t\left(\mathbf{c m o l c} / \mathbf{d m}^{3}\right)$ & $-0,18^{\text {ns }}$ & $0,45^{\mathrm{ns}}$ & $0,03^{\text {ns }}$ & $0,02^{\mathrm{ns}}$ & $-0,13^{\text {ns }}$ & $0,00^{\mathrm{ns}}$ \\
\hline $\mathbf{T}\left(\mathbf{c m o l c} / \mathbf{d m}^{3}\right)$ & $-0,25^{\text {ns }}$ & $-0,32^{\mathrm{ns}}$ & $-0,06^{\mathrm{ns}}$ & $0,50^{\mathrm{ns}}$ & $0,40^{\mathrm{ns}}$ & $0,04^{\mathrm{ns}}$ \\
\hline m $(\%)$ & $-0,20^{\mathrm{ns}}$ & $0,02^{\mathrm{ns}}$ & $0,01^{\mathrm{ns}}$ & $0,18^{\mathrm{ns}}$ & $0,03^{\mathrm{ns}}$ & $-0,12^{\mathrm{ns}}$ \\
\hline M.O. (dag/kg) & $0,04^{\mathrm{ns}}$ & $-0,01^{\mathrm{ns}}$ & $0,25^{\mathrm{ns}}$ & $0,30^{\mathrm{ns}}$ & $0,14^{\mathrm{ns}}$ & $-0,14^{\mathrm{ns}}$ \\
\hline Areia $(\mathbf{d a g} / \mathbf{k g})$ & $0,20^{\mathrm{ns}}$ & $0,15^{\mathrm{ns}}$ & $0,02^{\mathrm{ns}}$ & $-0,03^{\text {ns }}$ & $0,26^{\mathrm{ns}}$ & $-0,18^{\mathrm{ns}}$ \\
\hline Silte $(\mathrm{dag} / \mathbf{k g})$ & $-0,36^{\text {ns }}$ & $0,32^{\mathrm{ns}}$ & $-0,03^{\mathrm{ns}}$ & $-0,31^{\mathrm{ns}}$ & $-0,55^{\mathrm{ns}}$ & $0,22^{\mathrm{ns}}$ \\
\hline $\operatorname{Argila}(\mathbf{d a g} / \mathbf{k g})$ & $0,28^{\mathrm{ns}}$ & $-0,32^{\text {ns }}$ & $0,00^{\mathrm{ns}}$ & $0,32^{\mathrm{ns}}$ & $0,48^{\mathrm{ns}}$ & $-0,08^{\mathrm{ns}}$ \\
\hline Fogo & $-0,15^{\mathrm{ns}}$ & $-0,28^{\text {ns }}$ & -- & $-0,23^{\text {ns }}$ & $0,04^{\mathrm{ns}}$ & -- \\
\hline
\end{tabular}

Em que: Em que: CSS = Cerrado stricto sensu; $C D=$ Cerradão; FES = Floresta Estacional Semidecidual; $--=$ sem a variável fogo; $p=0,05 ;{ }^{n s}=$ não significativo; $\mathrm{P}=$ teor de fósforo disponível; $\mathrm{K}=$ teor de potássio disponível; $\mathrm{Ca}^{2+}=$ teor de cálcio disponível; $\mathrm{Mg}^{2+}=$ teor de magnésio disponível; $\mathrm{Al}^{3+}=$ teor de alumínio; $\mathrm{H}+\mathrm{Al}=$ acidez potencial; $\mathrm{SB}$ = soma de bases trocáveis; $\mathrm{t}$ = capacidade de troca de cátions efetiva; $\mathrm{T}$ = capacidade de troca de cátions a $\mathrm{pH} 7 ; \mathrm{m}=$ saturação por alumínio; V = saturação por bases; M.O. = matéria orgânica. 
Tabela 3 - Conclusão ...

Table 3 - Conclusion ...

b)

\begin{tabular}{|c|c|c|c|c|c|c|c|c|c|}
\hline \multirow{3}{*}{ Variáveis de solo } & \multicolumn{9}{|c|}{ Área basal por Fitofisionomia } \\
\hline & \multicolumn{3}{|c|}{ Recrutas } & \multicolumn{3}{|c|}{ Mortalidade } & \multicolumn{3}{|c|}{ Incremento } \\
\hline & CSS & CD & FES & CSS & CD & FES & CSS & CD & FES \\
\hline V (\%) & $0,37^{\text {ns }}$ & $0,43^{\text {ns }}$ & $0,16^{\mathrm{ns}}$ & $-0,39^{\text {ns }}$ & $-0,69^{*}$ & $-0,10^{\mathrm{ns}}$ & $-0,60^{*}$ & $0,14^{\mathrm{ns}}$ & $0,37^{\mathrm{ns}}$ \\
\hline $\mathbf{P}\left(\mathbf{m g} / \mathbf{d m}^{3}\right)$ & $0,18^{\mathrm{ns}}$ & $0,13^{\mathrm{ns}}$ & $0,40^{*}$ & $0,00^{\text {ns }}$ & $0,18^{\mathrm{ns}}$ & $-0,34^{\mathrm{ns}}$ & $0,45^{\mathrm{ns}}$ & $-0,13^{\mathrm{ns}}$ & $0,23^{\text {ns }}$ \\
\hline $\mathbf{K}\left(\mathbf{m g} / \mathbf{d m}^{3}\right)$ & $-0,29^{\text {ns }}$ & $0,15^{\mathrm{ns}}$ & $0,15^{\mathrm{ns}}$ & $0,01^{\mathrm{ns}}$ & $-0,40^{\mathrm{ns}}$ & $-0,12^{\mathrm{ns}}$ & $-0,26^{\text {ns }}$ & $0,39^{\text {ns }}$ & $-0,19^{\text {ns }}$ \\
\hline $\mathrm{Ca}\left(\mathbf{c m o l c} / \mathbf{d m}^{3}\right)$ & $0,38^{\mathrm{ns}}$ & $-0,08^{\mathrm{ns}}$ & $0,06^{\mathrm{ns}}$ & $0,00^{\text {ns }}$ & $0,09^{\text {ns }}$ & $-0,18^{\mathrm{ns}}$ & $-0,09^{n s}$ & $0,03^{\text {ns }}$ & $0,31^{\mathrm{ns}}$ \\
\hline $\operatorname{Mg}\left(\mathbf{c m o l c} / \mathbf{d m}^{3}\right)$ & $-0,22^{\text {ns }}$ & $0,70^{*}$ & $-0,23^{\text {ns }}$ & $-0,02^{\text {ns }}$ & $-0,52^{\text {ns }}$ & $0,16^{\mathrm{ns}}$ & $-0,21^{\mathrm{ns}}$ & $-0,20^{\text {ns }}$ & $0,04^{\mathrm{ns}}$ \\
\hline Al $\left(\mathbf{c m o l c} / \mathbf{d m}^{3}\right)$ & $-0,32^{\text {ns }}$ & $0,20^{\mathrm{ns}}$ & $0,02^{\mathrm{ns}}$ & $0,25^{\mathrm{ns}}$ & $0,21^{\mathrm{ns}}$ & $-0,19^{\mathrm{ns}}$ & $0,58^{*}$ & $-0,25^{\mathrm{ns}}$ & $-0,19^{\mathrm{ns}}$ \\
\hline $\mathbf{H}+\mathbf{A l}\left(\mathbf{c m o l c} / \mathbf{d m}^{3}\right)$ & $-0,29^{\text {ns }}$ & $-0,39^{\text {ns }}$ & $-0,28^{\mathrm{ns}}$ & $0,48^{\mathrm{ns}}$ & $0,72^{*}$ & $0,09^{\text {ns }}$ & $0,77^{*}$ & $-0,29^{\text {ns }}$ & $-0,43^{*}$ \\
\hline SB $\left(\mathbf{c m o l c} / \mathbf{d m}^{3}\right)$ & $0,15^{\mathrm{ns}}$ & $0,31^{\mathrm{ns}}$ & $0,00^{\mathrm{ns}}$ & $0,00^{\text {ns }}$ & $-0,36^{\mathrm{ns}}$ & $-0,14^{\mathrm{ns}}$ & $-0,19^{\text {ns }}$ & $0,09^{\text {ns }}$ & $0,32^{\text {ns }}$ \\
\hline $\mathbf{t}\left(\mathbf{c m o l c} / \mathbf{d m}^{3}\right)$ & $-0,16^{\mathrm{ns}}$ & $0,46^{\mathrm{ns}}$ & $0,00^{\mathrm{ns}}$ & $0,23^{\text {ns }}$ & $0,09^{\text {ns }}$ & $-0,15^{\mathrm{ns}}$ & $0,36^{\mathrm{ns}}$ & $-0,24^{\mathrm{ns}}$ & $0,32^{\text {ns }}$ \\
\hline $\mathbf{T}\left(\mathbf{c m o l c} / \mathbf{d m}^{3}\right)$ & $-0,29^{\text {ns }}$ & $-0,37^{\mathrm{ns}}$ & $-0,11^{\mathrm{ns}}$ & $0,49^{\text {ns }}$ & $0,69^{*}$ & $-0,15^{\mathrm{ns}}$ & $0,77^{*}$ & $-0,29^{\text {ns }}$ & $0,24^{\mathrm{ns}}$ \\
\hline $\mathbf{m}(\%)$ & $-0,22^{\text {ns }}$ & $-0,04^{\mathrm{ns}}$ & $-0,01^{\mathrm{ns}}$ & $0,11^{\mathrm{ns}}$ & $0,25^{\mathrm{ns}}$ & $-0,13^{\mathrm{ns}}$ & $0,39^{\text {ns }}$ & $-0,15^{\mathrm{ns}}$ & $-0,20^{\mathrm{ns}}$ \\
\hline M.O. (dag/kg) & $0,00^{\mathrm{ns}}$ & $0,00^{\mathrm{ns}}$ & $0,23^{\mathrm{ns}}$ & $0,25^{\mathrm{ns}}$ & $0,17^{\mathrm{ns}}$ & $0,00^{\mathrm{ns}}$ & $0,46^{\mathrm{ns}}$ & $-0,19^{\text {ns }}$ & $0,04^{\mathrm{ns}}$ \\
\hline $\operatorname{Areia}(\mathrm{dag} / \mathrm{kg})$ & $0,19^{\text {ns }}$ & $0,12^{\mathrm{ns}}$ & $0,04^{\mathrm{ns}}$ & $-0,12^{\text {ns }}$ & $0,09^{\mathrm{ns}}$ & $-0,10^{\text {ns }}$ & $0,00^{\mathrm{ns}}$ & $0,60^{\text {ns }}$ & $0,05^{\text {ns }}$ \\
\hline Silte $(\mathrm{dag} / \mathbf{k g})$ & $-0,30^{\text {ns }}$ & $0,32^{\mathrm{ns}}$ & $-0,05^{\mathrm{ns}}$ & $-0,27^{\mathrm{ns}}$ & $-0,81^{*}$ & $0,08^{\mathrm{ns}}$ & $-0,77^{*}$ & $0,12^{\mathrm{ns}}$ & $0,50^{*}$ \\
\hline Argila $(\mathrm{dag} / \mathbf{k g})$ & $0,23^{\text {ns }}$ & $-0,31^{\mathrm{ns}}$ & $0,01^{\mathrm{ns}}$ & $0,31^{\mathrm{ns}}$ & $0,76^{*}$ & $0,00^{\mathrm{ns}}$ & $0,75^{*}$ & $-0,20^{\text {ns }}$ & $-0,47^{*}$ \\
\hline Fogo & $-0,15^{\mathrm{ns}}$ & $-0,24^{\mathrm{ns}}$ & -- & $-0,25^{\mathrm{ns}}$ & $-0,01^{\mathrm{ns}}$ & -- & $-0,08^{n s}$ & $-0,45^{\mathrm{ns}}$ & -- \\
\hline
\end{tabular}

Fonte: Autores (2017)

Em que: CSS = Cerrado stricto sensu; $C D=$ Cerradão; FES = Floresta Estacional Semidecidual; -- = sem a variável fogo; $p=0,05 ;{ }^{\text {ns }}=$ não significativo; $\mathrm{P}=$ teor de fósforo disponível; $\mathrm{K}=$ teor de potássio disponível; $\mathrm{Ca}^{2+}=$ teor de cálcio disponível; $\mathrm{Mg}^{2+}$ = teor de magnésio disponível; $\mathrm{Al}^{3+}=$ teor de alumínio; $\mathrm{H}+\mathrm{Al}=$ acidez potencial; $\mathrm{SB}=$ soma de bases trocáveis; $\mathrm{t}$ = capacidade de troca de cátions efetiva; $\mathrm{T}$ = capacidade de troca de cátions a $\mathrm{pH} 7 ; \mathrm{m}=$ saturação por alumínio; V = saturação por bases; M.O. = matéria orgânica.

No CD, as correlações entre indivíduos recrutas, mortos e variáveis do solo foram todas não significativas (Tabela 3). A área basal dos indivíduos recrutas foi positivamente correlacionada com magnésio, enquanto a área basal dos indivíduos mortos se correlacionou positivamente com acidez de potencial, capacidade de troca de cátions a pH 7,0 e argila. Já a saturação por bases e silte foi negativamente correlacionada (Tabela 3).

$\mathrm{Na} F E S$, o fósforo foi positivamente correlacionado com indivíduos recrutas e não se correlacionou com indivíduos mortos. Já a área basal dos recrutas foi positivamente correlacionada com fósforo e o crescimento em área basal foi positivamente correlacionado com silte e negativamente com acidez potencial e argila (Tabela 3).

As informações contidas na Tabela 3 mostram que, mesmo com altos valores de acidez comuns em ambientes de Cerrado, ocorreram mudanças nas fitofisionomias CSS e CD. Nos sítios mais empobrecidos, as mudanças foram mais intensas, seja pelo crescimento diamétrico (incremento) no CSS ou pela mortalidade no CD, isto é, nessas fitofisionomias, o crescimento diamétrico e a mortalidade de indivíduos são independentes da fertilidade do solo, enquanto na FES, ambiente com maior fertilidade e baixa acidez em relação ao CSS e CD, do ponto de 
vista matemático, o fósforo teve correlação positiva tanto na área basal quanto no número dos indivíduos recrutas (Tabela 3).

Por meio da análise de correlação, o fogo não se correlacionou com as mudanças estruturais do ponto de vista matemático, pode ser que o espaço entre os inventários (5 anos) no CSS e CD tenha sido suficiente para os indivíduos se recuperarem ou porque a estatística empregada não teve a sensibilidade para diagnosticar o efeito do fogo, uma vez que o fogo afeta mais a regeneração natural e indivíduos menores. Portanto, o intervalo de observação não foi satisfatório, possivelmente, em uma análise feita em intervalos menores (pós-incêndio), esses resultados poderiam indicar correlação entre o fogo e a mortalidade.

Portanto, a ocorrência de incêndios pode provocar a morte dos indivíduos a curto prazo, de forma gradativa, ou até a recuperação destes, isso depende da intensidade, do grau dos danos provocados pelo fogo, da resistência adquirida ao longo do tempo e da idade fisiológica de cada indivíduo, por isso é importante estudos com intervalos menores. Pedroni (2001) notou que, se o intervalo entre os inventários for grande, maior será a dificuldade na interpretação dos possíveis fatores causadores da mortalidade.

Por isso, é importante a continuidade dos inventários, como se manterá as fitofisionomias em uma possível ocorrência ou ausência dos distúrbios. Essas informações são fundamentais para que se possa inferir sobre ações de conservação.

\section{Conclusão}

Os resultados mostram que ocorreram mudanças na estrutura das três fitofisionomias, a mortalidade foi superior ao recrutamento, à redução do número de indivíduos e à área basal. As mudanças foram maiores nas fitofisionomias CSS e CD atingidas pelo fogo, isso pela alta taxa de mortalidade. Na FES, as mudanças foram equilibradas entre saída e inclusão de novos indivíduos (recrutas), possivelmente reflexo do avanço sucessional. No CSS e no CD, os indivíduos das primeiras classes diamétricas demonstraram ser mais sensíveis à ocorrência do incêndio, houve alta taxa de mortalidade. Na FES, a perda de indivíduos foi mais equilibrada entre as classes diamétricas. Mesmo com a perda de indivíduos, a área basal no segundo inventário foi maior no CSS, em relação ao CD. Na FES, a área basal praticamente se manteve em relação ao primeiro inventário. Mesmo com altos valores de acidez comum em ambientes de Cerrado, as correlações foram positivas para incremento diamétrico dos indivíduos sobreviventes no CSS e para mortalidade no CD. Por fim, na FES, nos sítios mais férteis em relação ao CSS e CD, ocorreu aumento na área basal e no número de indivíduos. O CSS e CD estão se recuperando após distúrbio (fogo), com surgimento de novos indivíduos (recrutas) e crescimento diamétrico dos sobreviventes, porém o intervalo de 5 anos não foi suficiente para recuperação do número de indivíduos, enquanto na FES, sem distúrbio do fogo, a saída e a entrada de indivíduos foram mais equilibradas.

\section{Referências}

ALMEIDA, R. F. et al. Mudanças florísticas e estruturais no cerrado sensu stricto ao longo de 27 anos (1985-2012) na Fazenda Água Limpa, Brasília, DF. Rodriguésia, Rio de Janeiro, v. 65, n. 1, p. 1-19, jan./mar.2014.

ANGIOSPERM PHYLOGENY GROUP IV. An update of the Angiosperm Phylogeny Group classification for the orders and families of flowering plants: APG IV. Botanical Journal of the Linnean Society, [s. l.], v. 181, p. 1-20, 2016.

APPOLINÁRIO, V.; OLIVEIRA-FILHO, A. T.; GUILHERME, F. A. G. Tree population and community dynamics in a Brazilian tropical semideciduous forest. Revista Brasileira de Botânica, São Paulo, v. 28 n. 2, p. 347-360, abr./jun. 2005. 
AYRES, M. et al. BioEstat 5.3: aplicações estatísticas nas áreas das Ciências Biomédicas. Belém: Sociedade Civil Mamirauá, 2007. 324 p.

CHAZDON, R. L. et al. Rates of change in tree communities of secundar Neotropical forests following major disturbances. Philosophical Transactions of the Royal Society B-Biological Sciences, London, v. 362, p. 273-289, 2007.

DELCAMP, M. et al. Can functional classification of tropical trees predict population dynamics after disturbance? Journal of Vegetation Science, [s. l.], v. 19, p. 209-220, 2008.

EMBRAPA. Manual de métodos de análise de solo. 2. ed. Rio de Janeiro,1997.

EMBRAPA. Mapeamento de solos e aptidão agrícola das terras do Estado de Minas Gerais. Rio de Janeiro: Centro Nacional de Pesquisa de Solos, 2004.

FIGUEIREDO, L. T. M. et al. Alterações florísticas em uma floresta estacional semidecidual no município de viçosa - MG entre 1994 e 2008. Floresta, Curitiba, v. 43, n. 2, p. 169-180, abr./jun. 2013.

FRANCO, S. A. P. Estrutura e volume de povoamento de um remanescente de floresta estacional semidecidual em curvelo, MG.2012. Dissertação (Mestrado em Ciência Florestal) Universidade Federal dos Vales do Jequitinhonha e Mucuri, Diamantina, 2012.

HIGUCHI, P. et al. Dinâmica da comunidade arbórea em um fragmento de floresta estacional semidecidual montana em Lavras, Minas Gerais, em diferentes classes de solos. Revista Árvore, Viçosa, MG, v. 32, p. 417-426, 2008.

HOFFMANN, W. A. et al. Ecological thresholds at the savanna-forest boundary: how plant traits, resources and fire govern the distribution of tropical biomes. Ecology Letters, [s. l.], v. 15, n. 7, p. 759-768, jul. 2012.

HOFFMANN, W. A. et al. Tree top kill, not mortality, governs the dynamics of savana-forest boundairesunder frequent fire in central Brazil. Ecology, Washington, v. 90, p. 1326-1337, may 2009.

IVANAUSKAS, N. M.; MONTEIRO, R.; RODRIGUES, R. R. Alterations following a fire in a Forest community of Alto Rio Xingu (Mato Grosso, Brazil). Forest Ecology and Management, Amsterdam, v. 184, p. 239-250, out. 2003.

KLINK, C. A.; MACHADO, R. B. Conservation of the Brazilian Cerrado. Conservation Biology, [s. l.], v. 19, p. 707-713, 2005.

KORNING, J.; BALSLEV, H. Growth and mortality of trees in Amazonian tropical rain forest in Ecuador. Journal of Vegetation Science, [s. l.], v. 4, n. 1, p. 77-86, fev. 1994.

LIEBERMAN, D. et al. Mortality patterns and stand turnover rates in a wet tropical forest in Costa Rica. Journal of Ecology, Oxford, v. 73, n. 5, p. 915-924, nov. 1985.

LOPES, S. F.; VALE, V. S.; SHIAVINI, I. Efeito de queimadas sobre a estrutura e composição da comunidade vegetal lenhosa do cerrado sentido restrito em Caldas Novas, GO. Revista Árvore, Viçosa, MG, v. 33, n. 4, p. 695-704, maio 2009.

MACHADO, E. L. M.; OLIVEIRA-FILHO, A. T. Os padrões espaciais da dinâmica da comunidade arbórea são detectáveis em um fragmento pequeno (4 ha) e perturbado da Mata Atlântica brasileira. Acta Botanica Brasilica, São Paulo, v. 24, n. 1, p. 250-261, jan./mar. 2010.

MAGALHÃES, J. H. R.et al. Dinâmica do estrato arbóreo em uma floresta estacional semidecidual em Uberlândia, Minas Gerais, Brasil. Iheringia, Porto Alegre, v. 72, n. 3, p. 394-402, dez. 2017.

MEWS, H. A. et al. Dinâmica da comunidade lenhosa de cerrado típico na região nordeste do estado de Mato Grosso, Brasil. Biota Neotropica, Campinas, v. 11, n. 1, p. 73-82, jan. 2011. 
OLIVEIRA-FILHO, A. T.; MELLO, J. M.; SCOLFORO, J. R. S. Efeitos de perturbações e bordas passadas na estrutura e dinâmica da comunidade arbórea dentro de um fragmento de floresta tropical semidecídua no sudeste do Brasil durante um período de cinco anos (1987-1992). PlantEcology, [s. l.], v. 131, n. 1, p. 45-66, jul. 1997.

OTONI, T. J. O. et al. Componente arbóreo, estrutura fitossociológica e relações ambientais em um remanescente de cerradão, em Curvelo - MG. Cerne, Lavras, v. 19, n. 2, p. 201-211, abr./jun. 2013.

PEDRONI, F. Aspectos da estrutura e dinâmica da comunidade arbórea na Mata Atlântica e planície e encosta em Ubatuba, SP.2001. Tese (Doutorado em Biologia Vegetal) - Universidade Estadual de Campinas, Campinas, 2001.

PIVELLO, V. R. The use of fire in the Cerrado and Amazonian rainforests of Brazil: past and present. Fire ecology, [s. l.], v. 7, n. 1, p. 24-39, abr. 2011.

RIBEIRO, M. N. et al. Fogo e dinâmica da comunidade lenhosa em cerrado sentido restrito, Barra do Garças, Mato Grosso. Acta Botanica Brasilica, Belo Horizonte, v. 26, n. 1. p. 203-217, jul. 2012.

RIOS, M. N. S. et al. Mudanças pós-fogo na florística e estrutura da vegetação arbóreo arbustiva de um cerrado sentido restrito em Planaltina - DF. Ciência Florestal, Santa Maria, v. 28, n. 2, p. 469-482, abr.jun. 2018.

SATO, M. N.; GARDA, A. A.; MIRANDA, H. S. Fire effects in the mortality rate of woody vegetation in Central Brazil. In: INTERNATIONAL CONFERENCE ON FOREST FIRES RESEARCH, 3., 1998, Coimbra. Proceedings [...]. Coimbra: [s. n.], 1998. p. 1777-1784.

SHEIL, D.; JENNINGS, S.; SAVILL, P. Long-term permanent plot observations of vegetation dynamics in Budongo, a Ugandan rain forest. Journal of Tropical Ecology, Cambridge, v. 16, n. 6, p. 765-800, mar. 2000.

SILVA NETO, A. et al. Dinâmica da comunidade arbórea em um fragmento de cerrado Sensu Stricto em Minas Gerais, Brasil. Scientia Forestalis, Piracicaba, v. 45, n. 113, p. 21-29, mar. 2017.

SILVA, M. R.; ARAÚJO, G. M. Dinâmica da comunidade arbórea de uma floresta semidecidual em Uberlândia, MG, Brasil. Acta Botanica Brasilica, Brasília, v. 23, n. 1, p. 49-56, 2009.

STRAHLER, A.; STRAHLER, A. N. Physical geography: science and systems of the human 472 environment. 2nd ed. New York: John Wiley e Sons, 2002. 748 p.

VASCONCELOS, P. B.; ARAÚJO, G. M.; BRUNA, E. M. The role of roadsides in conserving Cerrado plant diversity. Biodiversity and Conservation, [s. l.], v. 23, n. 12, p. 3035-3050, 2014. 